\title{
PHASE TRANSFORMATIONS FOR CYCLOHEXANE IN MESOPOROUS SILICAS
}

\author{
JOHN DORE *, BEAU WEBBER *, JOHN STRANGE *, HOSSEIN FARMAN ${ }^{\dagger}$, \\ MARC DESCAMPS ${ }^{\ddagger}$ AND LAURENT CARPENTIER ${ }^{\ddagger}$ \\ ${ }^{*}$ Physics Lab.,University of Kent, Canterbury, CT2 7NR, UK \\ ${ }^{\dagger}$ Iran University of Science and Technology, Narmak, Iran \\ ${ }^{\ddagger}$ Physique, University of Science and Technology, Lille, France
}

\begin{abstract}
.
Neutron diffraction, NMR and DSC studies have been used to study the structural features of cyclohexane in the confined geometry of several mesoporous silicas with a pore size in the range $25-500 \AA$. The results show a consistent depression of the nucleation temperature that is inversely proportional to the pore size and appear to be related to the orientational correlations in the various phases. In the extreme case of MCM silicas, there is evidence that nucleation is completely suppressed.
\end{abstract}

\section{Introduction}

Cyclohexane $\mathrm{C}_{6} \mathrm{H}_{12}$ is an organic compound that comprises a ring molecule with a six-fold symmetrical structure. It is a liquid at room temperature but nucleates at $6.5^{\circ} \mathrm{C}[280 \mathrm{~K}]$ to form a plastic crystal phase that consists of a rotationally-disordered fcc lattice based on random orientation of the molecules. At lower temperature [196K], there is a further transition in which the rotation is effectively frozen out to give a brittle crystal phase. The molecule was extensively studied in the 1960's as a good example of a free rotator phase for an orientationally-disordered crystal. More recent work has shown that orientational correlations exist in both the liquid and plastic crystal phases and that there are significant variations in the structural characteristics as a function of temperature and pressure [1]. However, a detailed investigation of the features using computer simulation has not been carried out. 
The properties are particularly interesting when the cyclohexane is contained in the matrix of a porous solid as the transition temperatures are strongly affected by the pore dimensions. The depression of the nucleation point is approximately three times larger than that for water and is probably the largest for any molecular system that is liquid at room temperature. This effect is put to direct use in the measurement of pore distribution functions by the technique of NMR porosimetry $[2,3]$. The present paper describes the study of cyclohexane in various mesoporous silicas by the techniques of neutron diffraction [ND], nuclear magnetic resonance [NMR], cryoporimetry and differential scanning calorimetry [DSC].

\section{Theoretical Formalism and Experimental Technique}

\subsection{NEUTRON DIFFRACTION}

Neutron diffraction measurements on bulk $\mathrm{C}_{6} \mathrm{D}_{12}$ cyclohexane at various temperatures have already been reported $[4,5]$. The measured structure factor $\mathrm{S}_{M}(\mathrm{Q})$ may be written in terms of the intra- and inter-molecular terms, $\mathrm{f}_{1}(\mathrm{Q})$ and $\mathrm{D}_{M}(\mathrm{Q})$, respectively. The b-values for carbon and deuterium are approximately equal so all atoms make an equal contribution to the scattering and the composite pair correlation function $g(r)$ may be written as

$$
g(r)=0.111 g_{C C}(r)+0.445 g_{C D}(r)+0.444 g_{D D}(r)
$$

where $g_{\alpha \beta}(r)$ are the partial functions; the spatial distribution function can be evaluated from the Fourier transform of the interference terms in the diffraction pattern.

The diffraction profile is characterised by a relatively sharp peak at $1.2 \AA^{-1}$ followed by an oscillatory pattern that is characteristic of the molecular form-factor, $f_{1}(Q)$. The change from the liquid to the plastic crystal phase shows only a minor variation with the emergence of a few lowintensity Bragg peaks at intermediate Q-values, typical of a plastic crystal. At lower temperatures, the brittle crystal gives a more complex pattern with many sharp peaks.

Neutron diffraction measurements have been made of $\mathrm{C}_{6} \mathrm{D}_{12}$ in a samples of sol-gel and MCM silicas with nominal pore diameters of 100 and $30 \AA$, using the D4 diffractometer on the reactor at the Institut Laue Langevin, Grenoble. The results for the sol-gel silica, after subtraction of the silica substrate and container scattering are shown in Fig. 1 for a sequence of temperatures and are discussed in Sec3a; a detailed discussion of the results is given elsewhere [4-6]. 


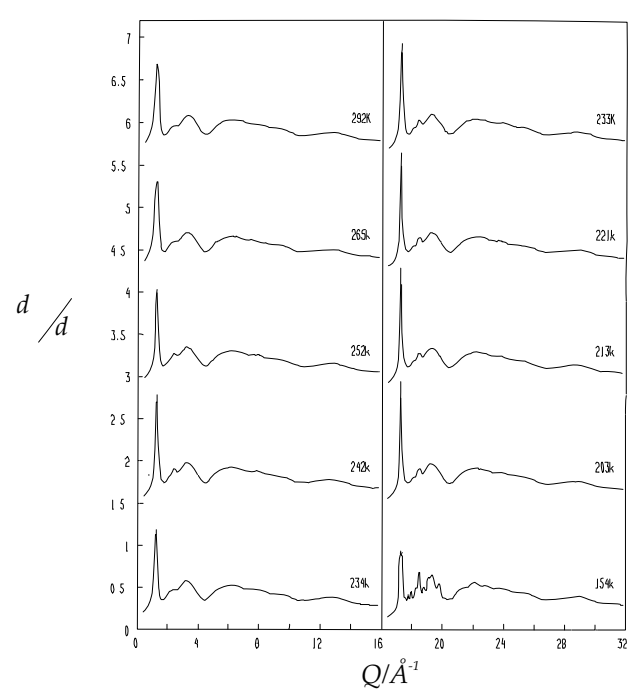

Figure 1. The neutron diffraction patterns for $\mathrm{C}_{6} \mathrm{D}_{12}$ in $100 \AA$ sol-gel silica as a function of temperature.

\subsection{NUCLEAR MAGNETIC RESONANCE}

Crystallites in the confined geometry of a mesoporous solid are known to melt at lower temperatures than the bulk material For NMR measurements it is convenient to study the 'plastic crystal to liquid' transition which occurs at a higher temperature than the 'liquid to plastic crystal'; this hysteresis effect is well known for these systems. For $\mathrm{C}_{6} \mathrm{H}_{12}$ [and water] in silicas of varying pore size, the melting curve has been measured as a function of temperature using over-filled samples. The NMR measurement effectively determines the amount of liquid within the pore volume as it is warmed. The cyclohexane on the outside of the silica granules behaves as bulk cyclohexane and provides a reference point for the change in melting temperature arising from the confinement. The depression of the melting point has been shown to be inversely proportional to the pore size as determined by gas adsorption and the variation of the NMR signal with temperature therefore gives a measurement of the effective pore-size distribution function [2,3,7].

A typical curve for cyclohexane in a $100 \AA$ sol-gel silica is shown in Fig $2 \mathrm{a}$, with the corresponding pore distribution function given in Fig $2 \mathrm{~b}$ using a calibration constant obtained from comparison with gas-phase absorption measurements. The results for various silicas covering a range of $25-500 \AA$ are shown in Fig 3, confirming the inverse relationship. However, there are some discrepancies with SANS results for the smaller pore sizes that possibly arise from shape-dependent effects. The results are discussed in 


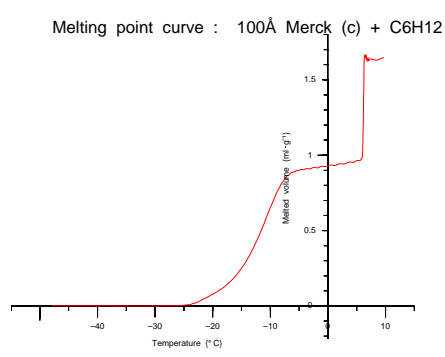

a. The NMR signal for $\mathrm{C}_{6} \mathrm{H}_{12}$ in a $100 \AA$ porous sol-gel silica.

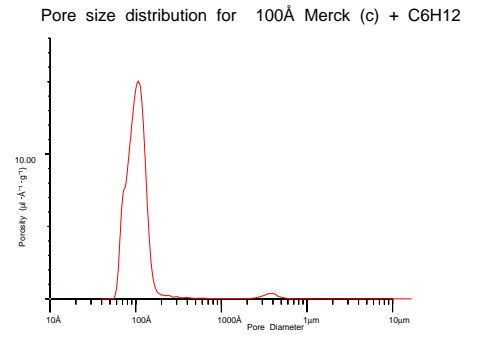

b. The corresponding pore distribution function.

Figure 2.

Melting point depression for $\mathrm{C}_{6} \mathrm{H}_{12}$ in $\mathrm{SiO}_{2}$ for $\tau=10 \mathrm{~ms}$

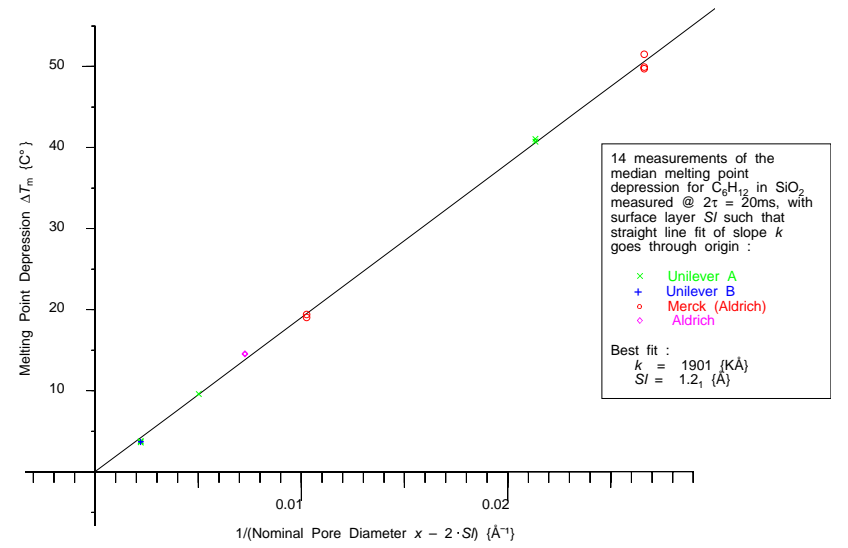

Figure 3. The relationship between the depression of nucleation point and inverse pore size for $\mathrm{C}_{6} \mathrm{H}_{12}$ in a series of sol-gel silicas.

Sec $3 b$.

\section{Experimental Results and Interpretation}

\subsection{NEUTRON DIFFRACTION FOR SOL-GEL SILICAS}

In $100 \AA$ silica, the 'liquid to plastic' crystal phase transition is depressed by $35 \mathrm{~K}$ and the brittle phase transition is also depressed by $20 \mathrm{~K}$. The sequence of measurements shown in Fig 1 indicates the state of the cyclohexane in a sample of $100 \AA$ sol-gel silica and confirms the deep super-cooling of the 
liquid before nucleation. There is a shift in the peak with temperature as would be expected from lattice contraction and a change in the profile of the main diffraction peak with little variation in height. A detailed analysis of the measurements $[4,5]$ shows that there is an associated change in density which appears to affect the orientational correlations in the confined geometry.

The inter-molecular potential for cyclohexane depends on a weak van der Waals interaction and is quite different from that for water, which has strong orientational dependence. Furthermore, the interaction with the silica interface will also be weak and non-directional, so the effects are primarily caused by the restricted geometry and the influence on the rotational freedom of the molecules in the confined state. The temperature of the phase transformation is therefore highly sensitive to the local environment but it is unclear why cyclohexane should show such a large effect compared with other molecular liquids of similar size and shape. In order to understand the phenomenon in more detail it will be necessary to conduct MD simulation studies to investigate the correlation of rotational and translational motion and how this coupling is influenced by local density and confined geometry.

\subsection{NMR CRYOPOROMETRY}

The NMR results enable the mean pore size to be evaluated for several different sol-gel silicas and, as shown in Fig 3, there is good agreement with the results from gas adsorption measurements. It is clear that the inverse relationship with pore size predicted by the Gibbs-Thomson theory is well obeyed over a large range of values. Models of extended pore systems were used to compare the NMR cryoporimetry and gas adsorption data with the neutron results, and these show surprising good agreement at large pore sizes, but there are some discrepancies with small angle neutron data for the lower pore sizes. This divergence at small pore sizes is not unexpected since it would be assumed that molecular size and the effects of pore shape would be expected to influence the behaviour on this scale. A critical comparison of these results will be presented separately [7].

\subsection{DIFFERENTIAL SCANNING CALORIMETRY}

DSC can be used to determine the heat flow during temperature change and provides a signature of phase changes through endo- or exo-thermic events. The use of modulated DSC is also useful in separating reversible and nonreversible changes [8]. The direct measurements for $\mathrm{C}_{6} \mathrm{H}_{12}$ confirmed the depression of nucleation point obtained from the NMR results and showed that there was no time-dependence in the data. This result showed that the 
effective pore distribution is large and confirmed that the response was not dependent on thermal cycling during the heating and cooling phase.

DSC heat flow for $\mathrm{C}_{6} \mathrm{H}_{12}$ in $100 \AA \mathrm{SiO}_{2}$

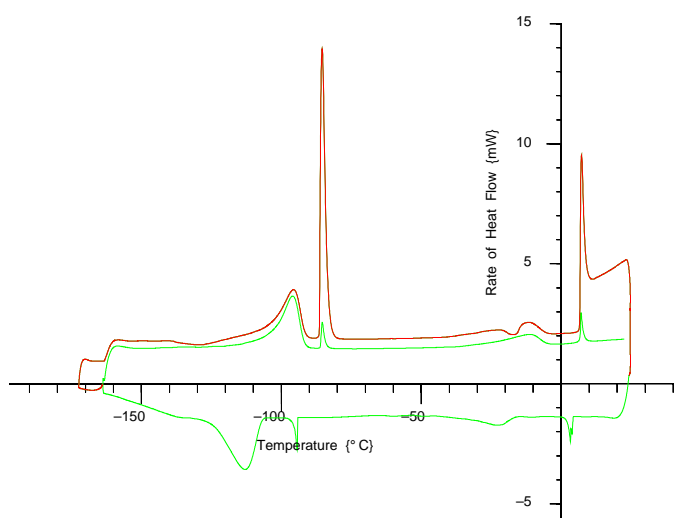

Figure 4. The DSC signal for $\mathrm{C}_{6} \mathrm{H}_{12}$ in a $100 \AA$ porous sol-gel silica for a double warming and single cooling cycle.

A separate set of measurements was also made for some samples, which had been rapidly cooled by quenching in liquid nitrogen. The results are shown in Fig 4 for an initial warming cycle followed by a cooling and rewarming sequence. The large endothermic peaks in the initial warming cycle arise from excess cyclohexane outside the pores, and correspond to the conventional bulk phase transitions from brittle crystal to plastic crystal at $186 \mathrm{~K}$ and from the plastic crystal to liquid at $280 \mathrm{~K}$. The weaker broad peaks at $176 \mathrm{~K}$ and $262 \mathrm{~K}$ correspond to the equivalent endothermic transition for the pore cyclohexane. An interesting feature of the curves is the two small exothermic peaks at $143 \mathrm{~K}$ and $255 \mathrm{~K}$ in the initial warming cycle. These broad peaks imply that the quenched sample has been formed in a metastable state but relaxation can only occur when sufficient thermal energy is provided to allow structural re-arrangement. Similar features are observed for cyclohexane in silica samples of different pore size. The peaks are not present in the second warming cycle and are therefore a feature of the quenched material.

These observations seem to indicate that the cyclohexane is in a disordered or glassy state analagous to that more frequently studied for fragile glass-forming systems such as OTP or TPP. It will be interesting to make diffraction measurements on the quenched state to check this conjecture. Corresponding NMR measurements are difficult to conduct due to the use of 
larger sample volumes and the difficulty of achieving a satisfactory quench in a sealed silica ampoule. Further studies are in progress.

\section{Conclusions}

The present results have demonstrated that a simple molecule such as cyclohexane exhibits a number of unusual characteristics when placed in the confined geometry of a mesoporous solid. The large change in the phase transformation temperatures is thought to be due to the coupling of translational and rotational dynamics arising from the restricted pore volume but quantitative explanation has not yet been achieved. The features shown in the DSC results for the quenched sample are also puzzling and will need further investigation. In small pores, it seems that the nucleation may be completely suppressed but further work will need to be undertaken to fully explore the structural characteristics of this state and to investigate possible links to the formalism used to describe the behaviour of a fragile glass system.

\section{Acknowledgements}

The present work has been conducted using neutron facilities at the ILL supported by the EPSRC and the recent joint work between UKC and USTL has been funded by a regional grant in the Interreg scheme supported by the European Community programme. One of us (HF) wishes to thank the Iranian Government for a travel grant.

\section{References}

1. H.Farman, F.Coveney, J.C.Dore \& M-C. Bellissent-Funel, Mol. Phys. 87, 1217-1233, (1996) and papers cited therein.

2. J.B.W.Webber, PhD thesis, Characterising Porous Media. University of Kent, (2000).

3. J.B.W.Webber, J.H.Strange \& J.C.Dore, in press, Mag. Res. Imag., 2001; paper presented at RAMRAPM conference, Bologna, (2000).

4. H.Farman, L. O'Mard, J.C. Dore \& M-C. Bellissent-Funel, Mol. Phys. 73, 855, (1991).

5. H.Farman, J.C. Dore \& J.B.W. Webber, in press, J.Mol.Liq., (2001); paper presented at the EMLG conference, Regensburg, (2000).

6. H.Farman \& J.C.Dore, submitted to Chem. Phys., (2001).

7. J.B.W.Webber, J.C.Dore \& J.H.Strange, to be submitted.

8. M.Descamps, J-F. Willart, private communication; papers in press. 